\title{
Author Correction: Transcriptomic and cellular decoding of regional brain vulnerability to neurogenetic disorders
}

Jakob Seidlitz (1), Ajay Nadig, Siyuan Liu, Richard A. I. Bethlehem, Petra E. Vértes, Sarah E. Morgan (1), František Váša, Rafael Romero-Garcia (D), François M. Lalonde (D), Liv S. Clasen, Jonathan D. Blumenthal, Casey Paquola (1), Boris Bernhardt, Konrad Wagstyl, Damon Polioudakis, Luis de la Torre-Ubieta, Daniel H. Geschwind (1), Joan C. Han, Nancy R. Lee, Declan G. Murphy (D, Edward T. Bullmore \& Armin Raznahan

Correction to: Nature Communications https://doi.org/10.1038/s41467-020-17051-5, published online 3 July 2020.

The original version of this Article contained an error in the "Results" section, which incorrectly read "For example, the most-extreme ranking CNV region gene relative to anatomical change in each CNV was; +X: ZCCHC12, +Y: EIF1AY, $-\mathrm{X}:$ GABRA3, $+21:$ PCP4, -22q11: MAPK1, -11p13: TRIM44." The correct version states "For example, the most extreme ranking CNV region gene relative to anatomical change in each CNV was: +X: ZCCHC12, +Y: EIF1AY, -X: GABRA3, +21: PCP4, -22q11: SLC7A4, -11p13: TRIM44."

The original version of this Article contained an error in the "Results" section, which incorrectly read "MS increases in VCFS syndrome (-22q11) and MAPK1 expressing inhibitory neurons." The correct version removes this sentence.

The original version of this Article contained an error in Figs. $1 \mathrm{~b}$ and $2 \mathrm{~d}$.

In the original version of Fig. $1 \mathrm{~b}$, the graph next to the Chr22 schematic reported the following: the $y$-axis range was $0 \mathrm{e}+00$ to $3 \mathrm{e}-04$, the $x$-axis range was -3000 to 3000 , and the values for the $P_{\text {RAND-Trans }}$ and $P_{\text {RAND-Cis }}$ were 0.0094 and 0.0018 , respectively. 
The correct version of Fig. 1 is:

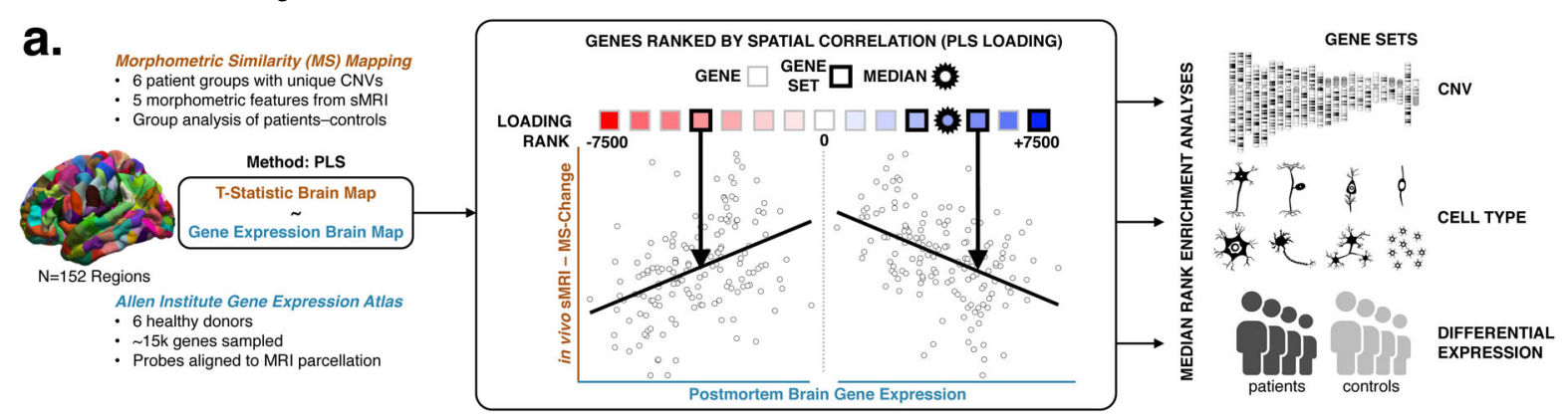

b.

Morphometric Similarity Change

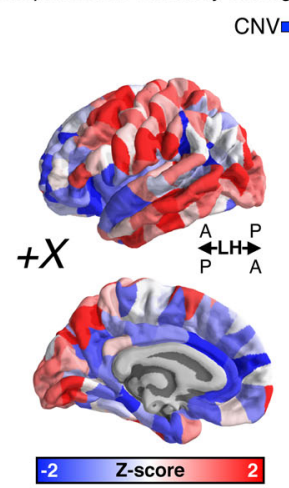

$\mathrm{CNV} \Rightarrow \mathrm{x}$
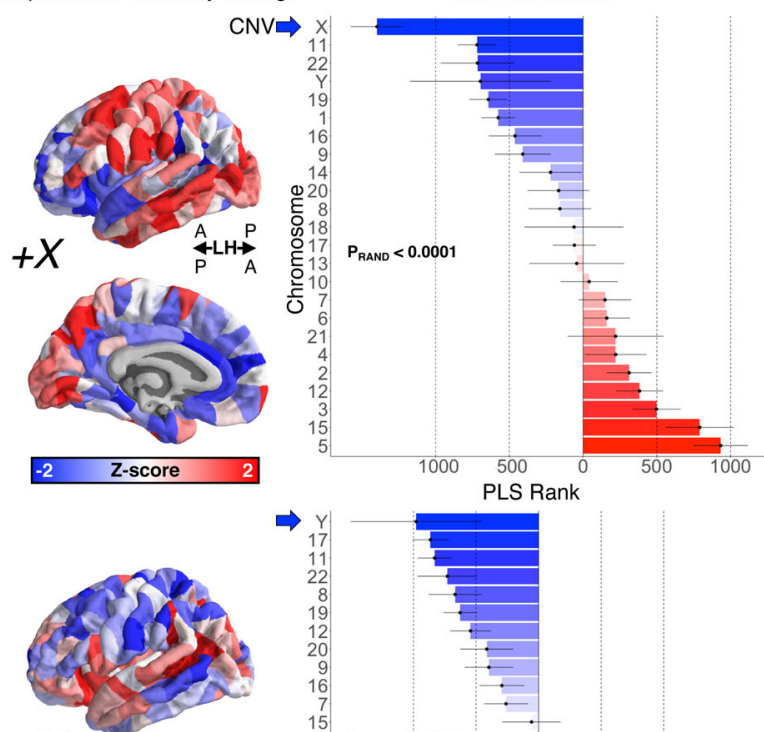

$+Y$
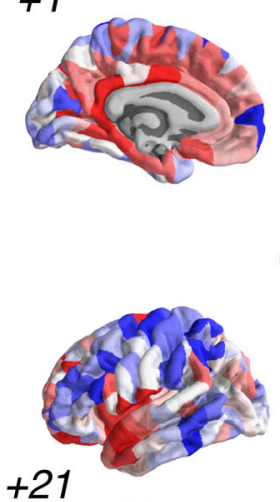

$+21$

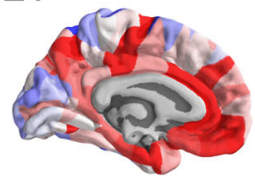

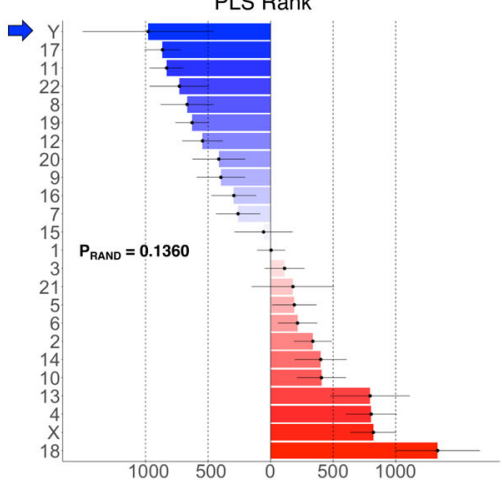

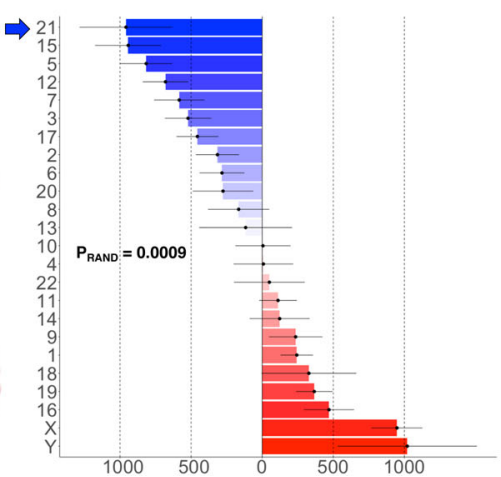

Morphometric Similarity Change
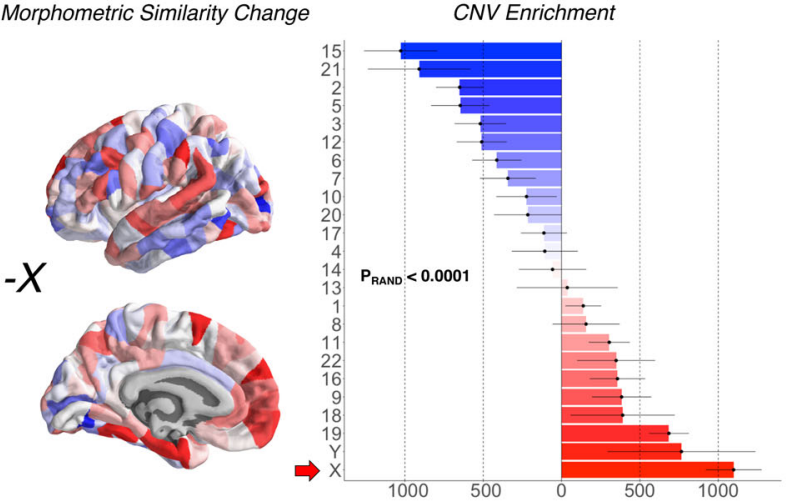

VCFS
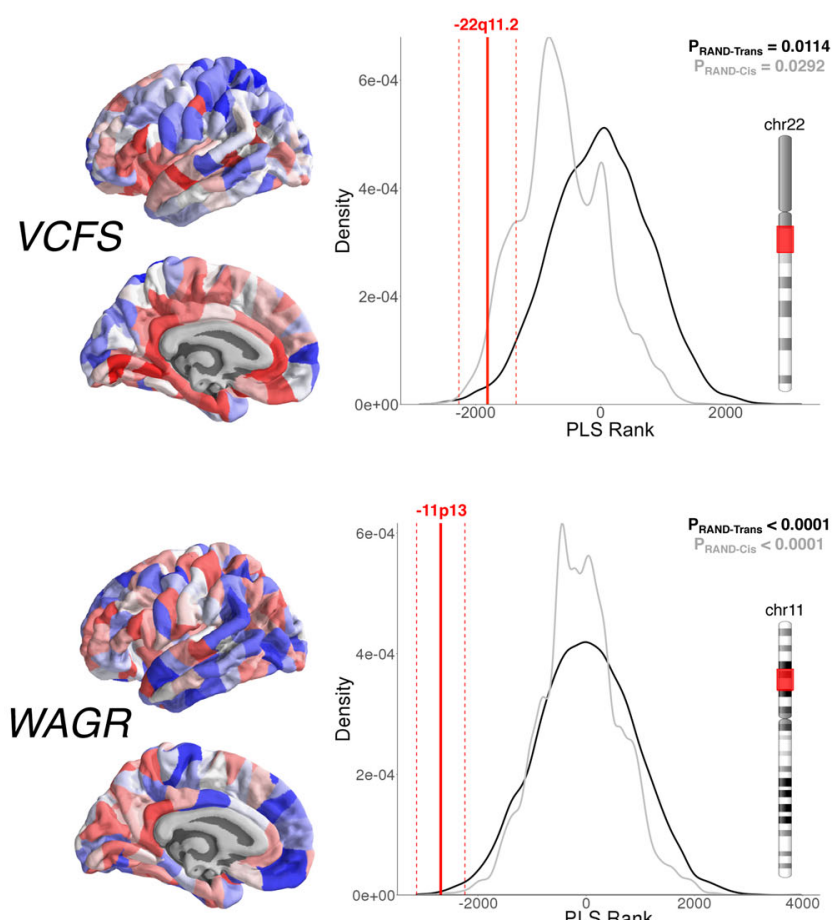
which replaces the previous incorrect version:

a

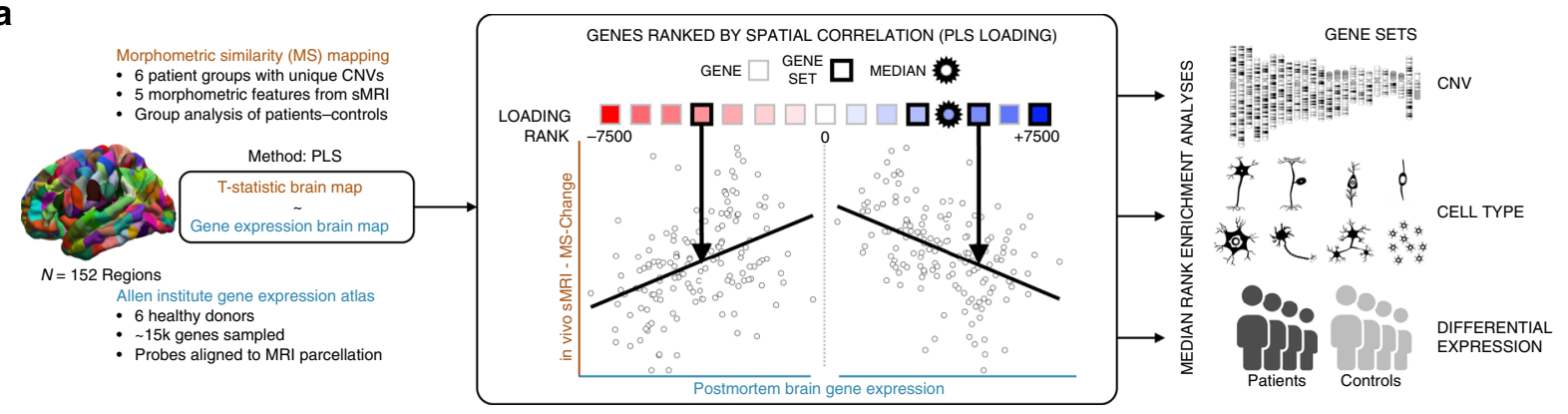

b Morphometric similarity change
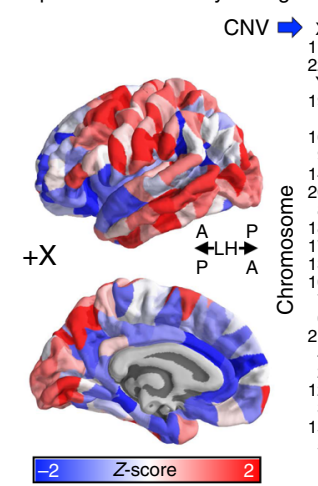

CNV enrichment
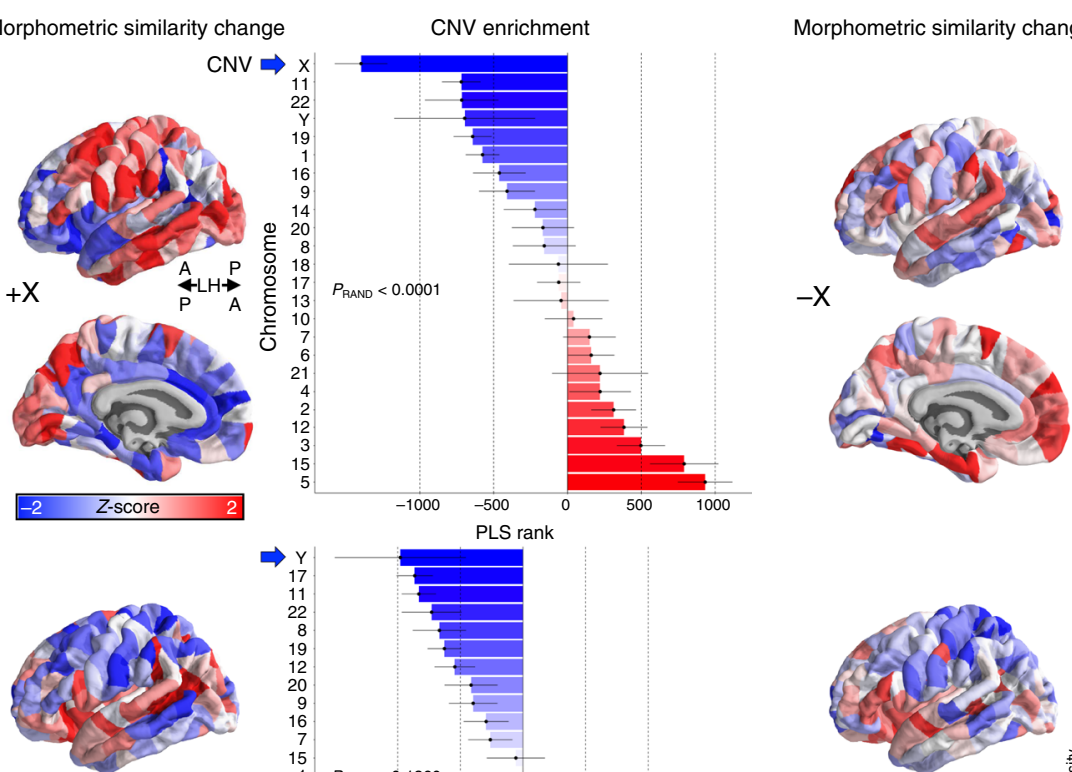

$+\mathrm{Y}$
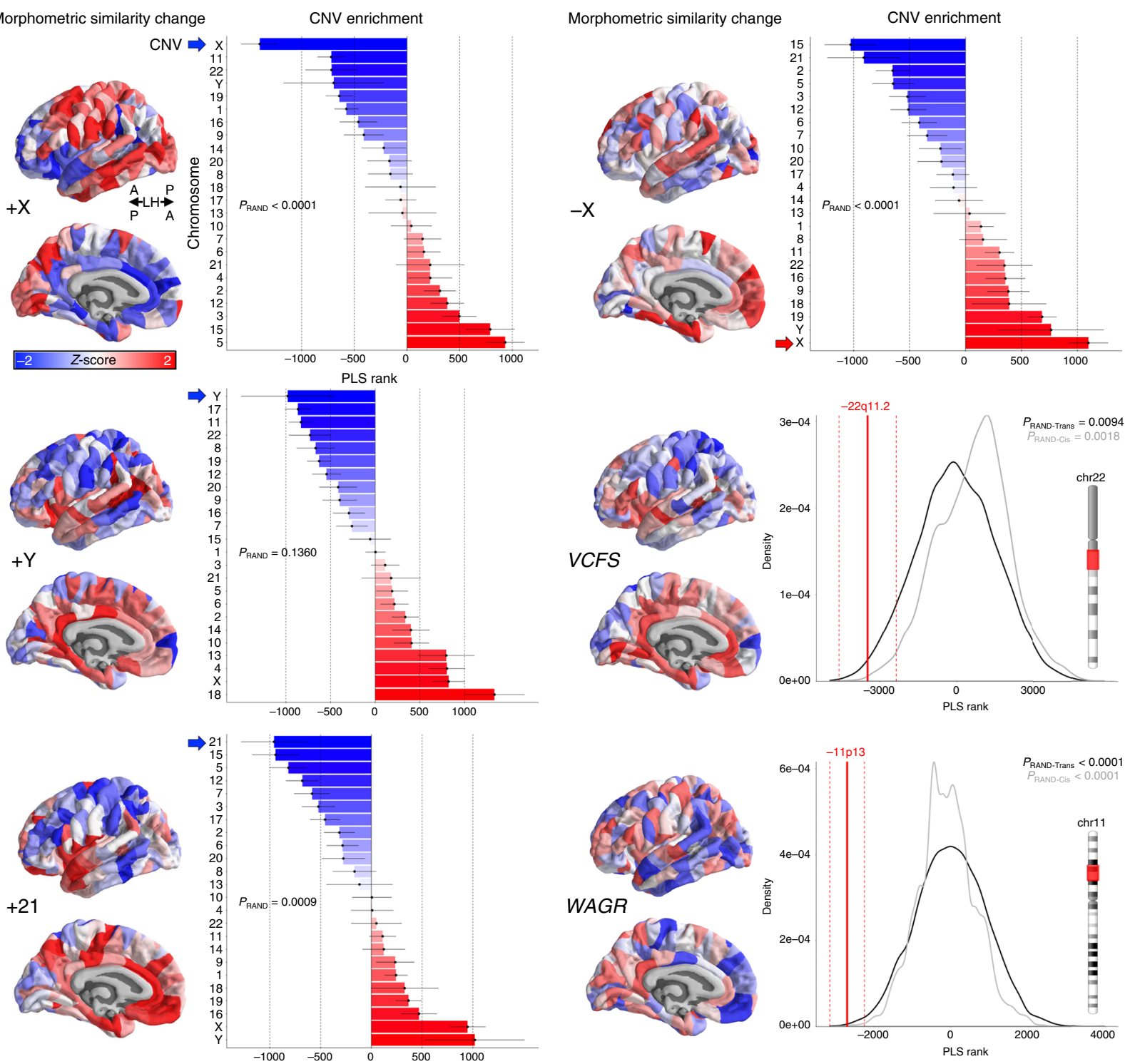

In the original version of Fig. $2 \mathrm{~d}$, the $y$-axis reported the following class of cells: OPC, Oligo, Neuro-In, Endo, Astro. Among the reported genes in the plot, MAPK1 is included. 
The correct version of Fig. 2 is:

a.

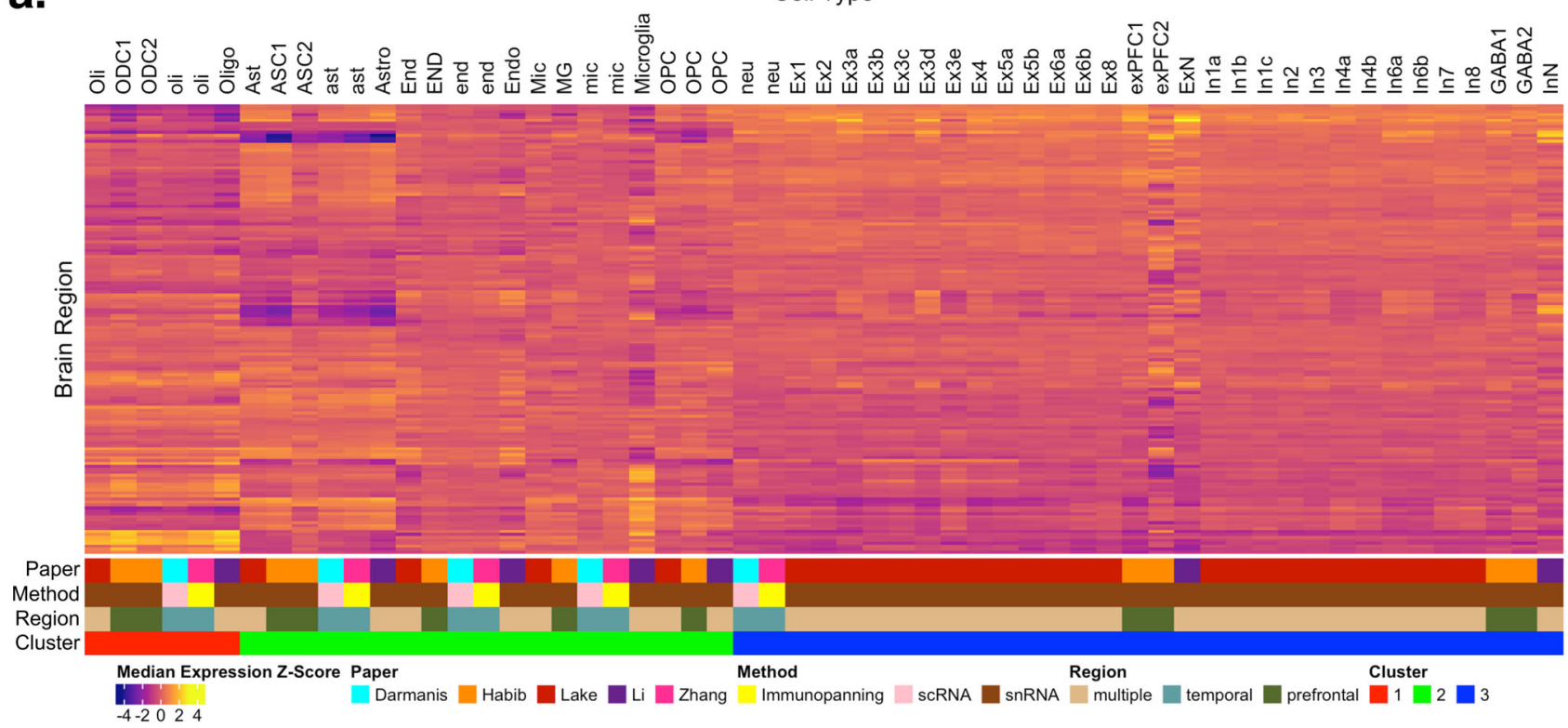

b.

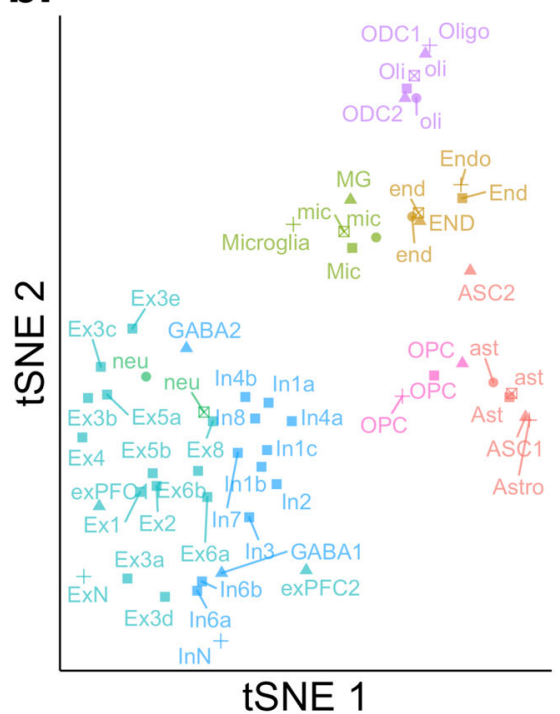

d.

OPC

Oligo

0
0
$\frac{\pi}{U}$

Endo

Astro

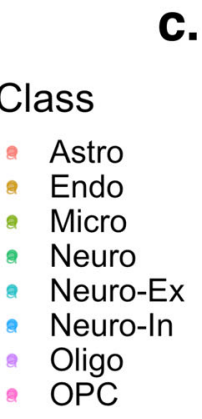

C.

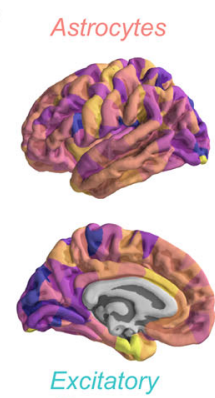

Paper

- Darmanis

- Habib

- Lake

$+\mathrm{Li}$

$\otimes$ Zhang

tSNE 1

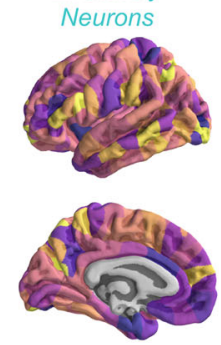

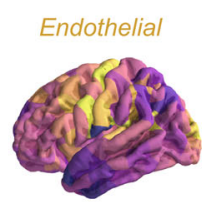
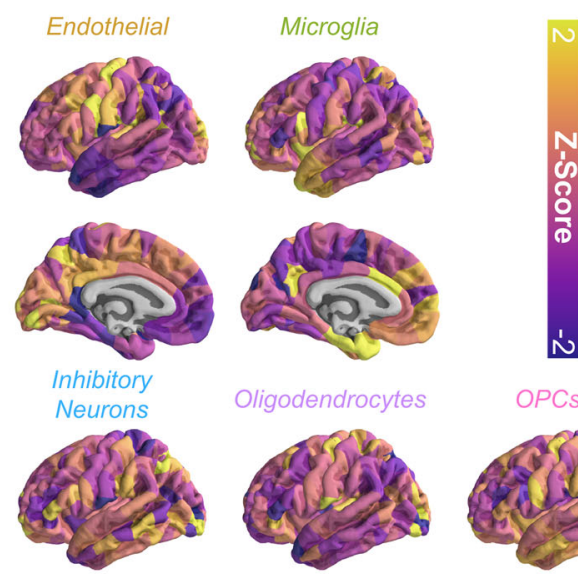

Oligodendrocytes
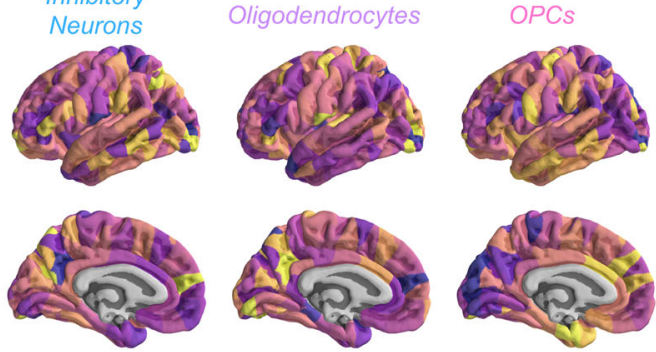

NCAM2

ENOX2

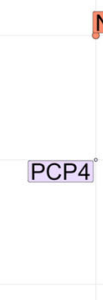

ITM2A

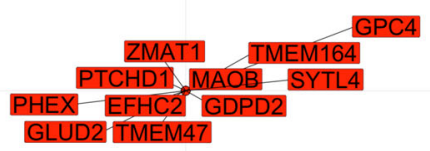

CNV 
which replaces the previous incorrect version:

a

Cell type

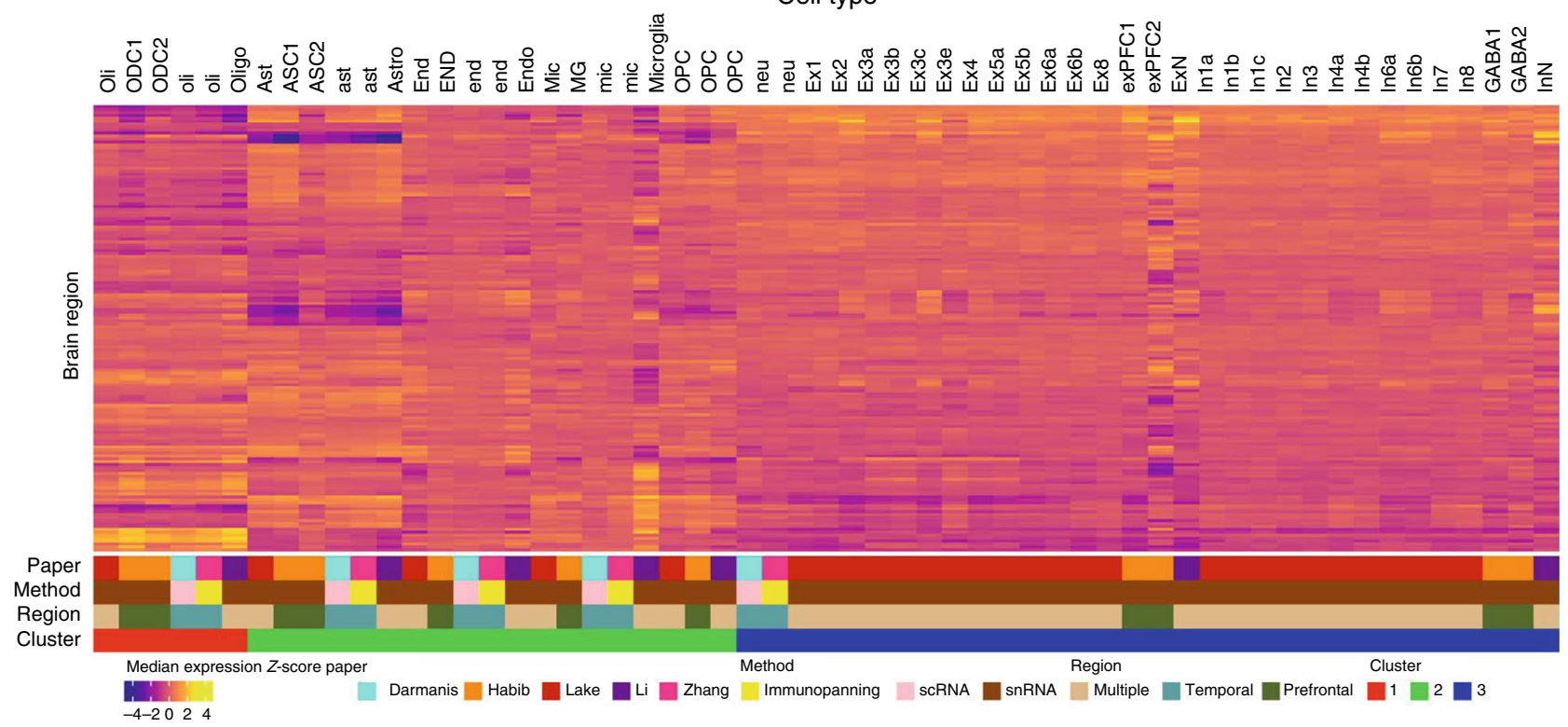

b

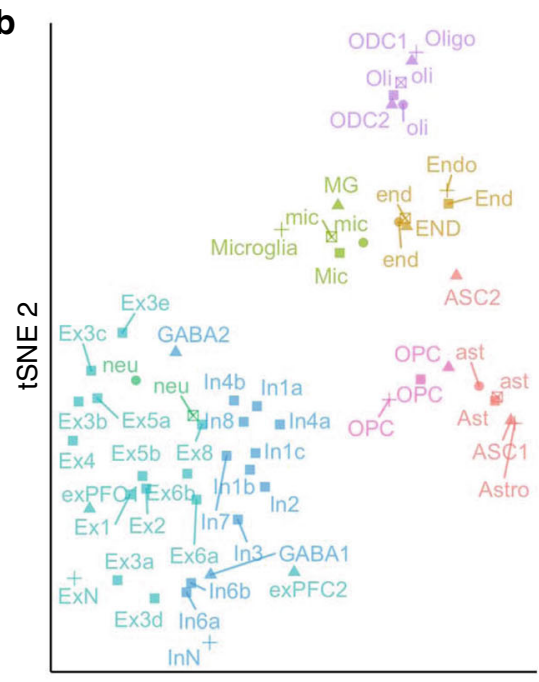

tSNE 1
Class

Astro

ando

Micro

2 Neuro

a Neuro-Ex

a Neuro-In

Oligo

OPC

Paper

- Darmanis

$\triangle$ Habib

- Lake

$+\mathrm{Li}$

$\otimes$ Zhang

c
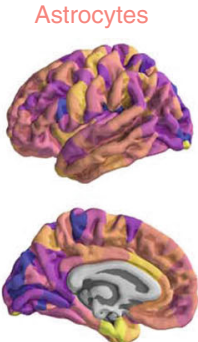

Excitatory

Neurons
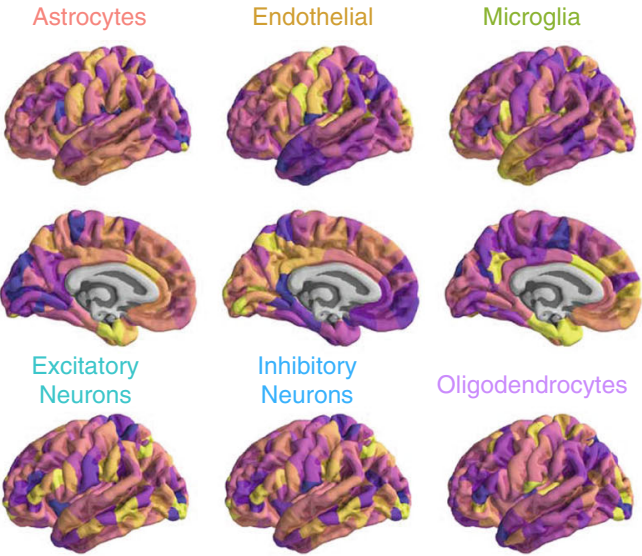

Inhibitory

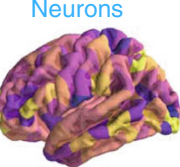

Oligodendrocytes
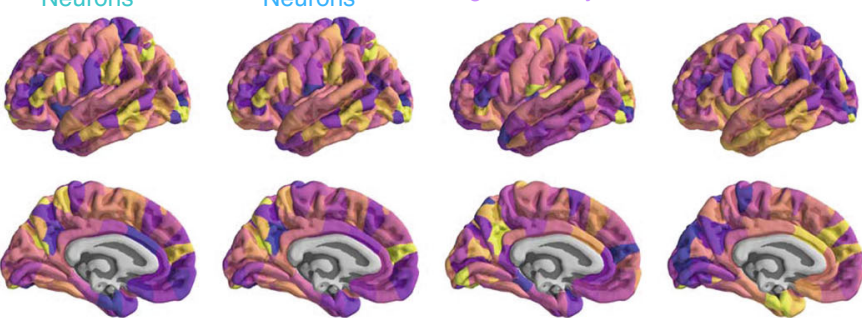

NR0B1

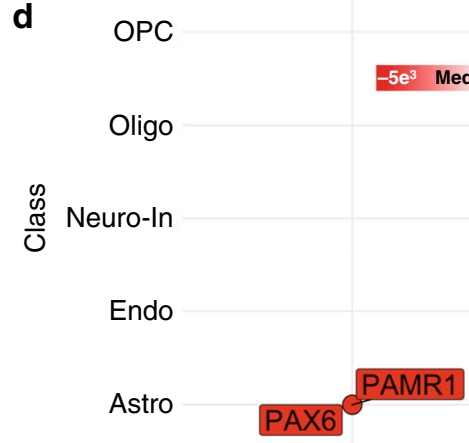

$-11 \mathrm{p} 13$
ENOX2 NCAM2 AMMECR1
AMMECR1

MAPK1
PCP4 
All the errors above have been corrected in both the PDF and HTML versions of the Article.

The original version of the Supplementary Information associated with this Article included an incorrect Supplementary Data 1 file, in which the CVN genes tab, column A (del22q11.2) reported 20 genes in total: DGCR6, PRODH, STK22B, ES2EL, GSCL, SLC25A1, ZDHHC8, RANBP1, HTF9C, DGCR8, T10, ARVCF, COMT, TBX1, GP1BB, UFD1L, HIRA, SNAP29, TMEM191A, MAPK1.

The HTML has been updated to include a corrected version of Supplementary Data 1; the original incorrect version of Supplementary Data 1 can be found as Supplementary Information associated with this Correction.

Published online: 17 November 2020

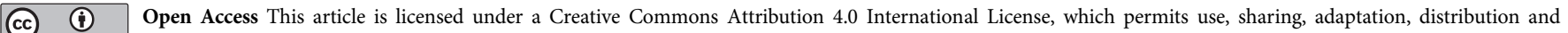
reproduction in any medium or format, as long as you give appropriate credit to the original author(s) and the source, provide a link to the Creative Commons license, and indicate if changes were made. The images or other third party material in this article are included in the article's Creative Commons license, unless indicated otherwise in a credit

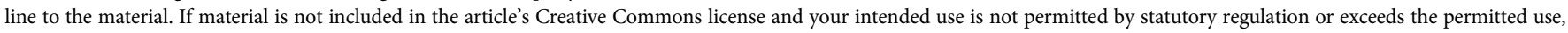
you will need to obtain permission directly from the copyright holder. To view a copy of this license, visit http://creativecommons.org/licenses/by/4.0/.

(C) The Author(s) 2020 